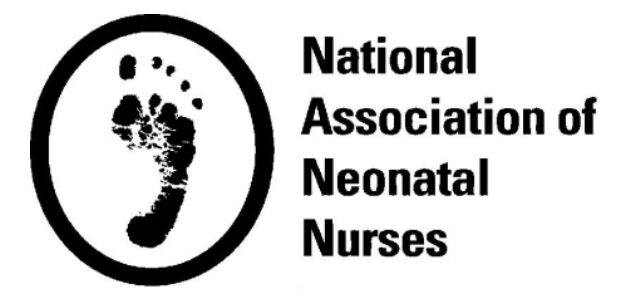

\title{
Racial Disparity in the NICU
}

\section{Position Statement}

\section{\#3070}

NANN Board of Directors

June 2020

\section{Introduction}

Preterm birth continues to be a significant public health problem in the United States. In 2018, one out of every 10 infants in the United States was born prior to 37 weeks gestation (Centers for Disease Control and Prevention, 2019). The U.S. preterm birth rate increased to $10.02 \%$ in 2018, representing a 1\% increase from 2017 and the fourth straight year of increases in this rate. The increase in the preterm birth rate among non-Hispanic White mothers between 2017 and 2018 was not statistically significant ( 9\%), but preterm delivery rates among non-Hispanic Black mothers increased to $14.13 \%$ and among Hispanic mothers to $9.73 \%$. (Martin, Hamilton, Osterman, \& Driscoll, 2019). These statistics are even more concerning given the racial health disparities for premature infants that have been well documented in the literature.

\section{Background}

There are numerous studies demonstrating health disparities among preterm infants in the neonatal intensive care unit (NICU), and the causes have been found to be multifactorial. Three different contributors to health disparities for neonates have been described in the literature (Beck et al., 2019):

- Increased risk for preterm birth. Maternal challenges such as lack of access to prenatal and interconception care; decreased interpregnancy intervals; and poorer social determinants of health, which can be defined as the conditions in the environments in which people live, learn, work, play, worship, and age, all affect the risk for preterm birth (U.S. Department of Health and Human Services, Office of Disease Prevention and Health Promotion, 2020).

- Lower quality of care. Infants from racial minority groups more often are born in institutions where the nurse-to-patient ratio is high. These NICUs also have a greater 
incidence of missed care activities, lower breastfeeding rates, and higher infection rates (Horbar et al., 2019). In addition, differences in the application of evidence-based practices for different racial and ethnic groups within individual units, and poorer resulting outcomes, have been noted (Profit et al., 2017).

- Socioeconomic disadvantages in infancy and childhood. The impact of social determinants of health not only contribute to preterm birth, but also influence the longterm health outcomes of NICU graduates (Beck et al., 2019).

Statistics show that Black infants have a death rate more than twice that of White or Hispanic infants, and there also is a higher rate of significant morbidities in the Black and Hispanic groups (Howell et al., 2018). Compared with White infants, Black infants are more likely to be delivered in hospitals with higher risk-adjusted mortality rates (Howell, Herbert, Chatergee, Klienman, \& Chassin, 2008; Howell, et al., 2018) and to receive care in NICUs with lower quality scores (Hobar et al., 2019). Even after accounting for NICU quality between geographic regions, the findings of segregation and inequality among very preterm and very low-birth-weight infants persisted. In addition, Hobar et al. found that compared with White infants, Asian infants received care in $\mathrm{NICUs}$ with higher quality scores.

\section{Conclusion}

The National Association of Neonatal Nurses (NANN) endorses healthcare equality for all infants regardless of race, parental economic or educational status, or geographic area.

\section{NANN's Recommendations}

1. Elevate awareness of racial disparities, inclusion, and cultural sensitivity by providing education in cultural competence, presenting published research on the issues, and having open discussions about the topics.

2. Encourage diversity in the workforce.

3. Examine personal bias and beliefs, some of which may be unconscious. Be self-aware and open to feedback and observations from others.

4. Examine individual NICU statistics to evaluate significant trends in gestational age, race, and patient outcomes.

5. Invite families to participate in the culture of the NICU by involving a diverse team of parents on committees, such as a quality improvement committee.

6. Regularly use interpreters when caring for families who do not speak English. Relying on other family members to interpret for parents may contribute to misinformation and a lack of appropriate education.

7. Provide written and electronic information in multiple languages whenever possible.

8. Consider all discharge requirements and available resources to transition families to the home environment.

9. Advocate for racial awareness and equality in your hospital and community. Connect with hospital administrators, community leaders, and elected officials to discuss health outcomes of racial disparities, and advocate for resources that positively impact the social determinates of health affecting maternal and infant health. 


\section{References}

Beck, A. F., Edwards, E. M., Horbar, J. D., Howell, E. A., McCormick, M. C., \& Pursley, D. M. (2019). The color of health: How racism, segregation, and inequality affect the health and well-being of preterm infants and their families. Pediatric Research, 87(2), 227-234. doi:10.1038/s41390-019-0513-6

Centers for Disease Control. (2019, October 21). Preterm birth. Retrieved March 8, 2020, from https://www.cdc.gov/reproductivehealth/maternalinfanthealth/pretermbirth.htm

Horbar, J. D., Edwards, E. M., Greenberg, L. T., Profit, J., Draper, D., Helkey, D., . . Firebaugh, G. (2019). Racial segregation and inequality in the neonatal intensive care unit for very low-birth-weight and very preterm infants. JAMA Pediatrics, 173(5), 455-461.

Howell, E., Hebert, P., Chatterjee, P., Kleinman, L., and Chassin, M. (2008). Black/ White differences in very low birth weight neonatal mortality rates among New York City hospitals. Pediatrics, 121(3): e407-e15.

Howell, E. A., Janevic, T., Hebert, P. L., Egorova, N. N., Balbierz, A., \& Zeitlin, J. (2018). Differences in morbidity and mortality rates in black, white, and Hispanic very preterm infants among New York City hospitals. JAMA Pediatrics, 172(3), 269-277.

Martin, J., Hamilton, B. E., Osterman, M. J. K., \& Driscoll, A. (2019) Division of vital statistics. Births: Final data for 2018, 68(13). Hyattsville, MD: National Center for Health Statistics. Retrieved March 8, 2020, from https://www.cdc.gov/nchs/data/nvsr/nvsr68/nvsr68 13508.pdf

Profit, J., Gould, J. B., Bennett, M., Goldstein, B. A., Draper, D., Phibbs, C. S., \& Lee, H. C. (2017). Racial/ethnic disparity in NICU quality of care delivery. Pediatrics, 140(3), e20170918. doi:10.1542/peds.2017-0918

U.S. Department of Health and Human Services, Office of Disease Prevention and Health Promotion. (2020). Social determinants of health. Retrieved March 9, 2020, from https://www.healthypeople.gov/2020/topics-objectives/topic/social-determinants-ofhealth

Copyright (C) 2020 by the National Association of Neonatal Nurses. No part of this statement may be reproduced without the written consent of the National Association of Neonatal Nurses.

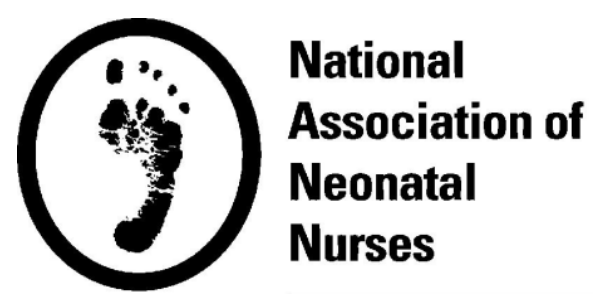

8735 W. Higgins Road, Chicago, IL 60631 $800.451 .3795 \cdot 847.375 .3660 \cdot$ Fax 866.927.5321 www.nann.org 